\title{
INDIVIDUO: results from a patient-centered lifestyle intervention for obesity surgery candidates
}

José Camolas $^{\mathrm{a}, \mathrm{b}}$, Osvaldo Santos ${ }^{\mathrm{c}}$, Pedro Moreira ${ }^{\mathrm{d}}$, Isabel do Carmo ${ }^{\mathrm{a}}$

${ }^{a}$ Hospital Santa Maria / Faculdade de Medicina da Universidade de Lisboa, Portugal

${ }^{\mathrm{b}}$ Instituto Superior de Ciências da Saúde Egas Moniz, Monte de Caparica, Portugal

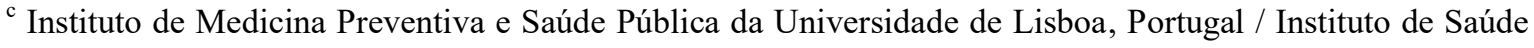
Ambiental da Universidade de Lisboa, Portugal

${ }^{\text {d }}$ Faculdade de Ciências da Nutrição e Alimentação da Universidade do Porto, Portugal

\section{Corresponding author at:}

José Camolas

Serviço de Endocrinologia, Diabetes e Metabolismo

Hospital Santa Maria, Piso 6

Av. Prof. Egas Moniz

1649-035 Lisboa

E-mail: jose.camolas@gmail.com

Telephone number: +351934596374 


\section{Abstract}

Background: Preoperative nutritional counseling provides an opportunity to ameliorate patients' clinical condition and build-up adequate habits and perception of competence. Study aimed to evaluate: (a) the effect of INDIVIDUO on weight and metabolic control; (b) the impact of INDIVIDUO on psychosocial variables associated with successful weight-control.

Methods: Two-arm randomized controlled single-site study, with six-month duration. Patients were recruited from an Obesity Treatment Unit's waiting list. For the intervention group (IG), an operating procedure manual was used, nutritionists received training/supervision regarding INDIVIDUO's procedures. Control group (CG) received literacy-promoting intervention. Intention-to-treat and per-control analysis were used. Outcomes included weight, metabolic control variables (blood pressure, glycaemia, insulinemia, triglycerides, cholesterol), measures of eating and physical activity patterns, hedonic hunger, autonomous/controlled regulation, perceived competence for diet (PCS-diet) and quality of life. Primary outcomes were weight and metabolic control. Effect size was estimated by odds ratio and Cohens'd coefficient.

Results: 94 patients participated (IG:45; CG:49) and 60 completed the study (IG:29; CG:31). Intervention patients lost an excess $9.68 \%$ body weight (\%EWL), versus $0.51 \%$ for CG. Adjusting for age and baseline $\mathrm{BMI}$, allocation group remained an independent predictor of $\% \mathrm{EWL}(\mathrm{B}=8.43,95 \% \mathrm{CI}$ : 2.79-14.06). IG had a six-fold higher probability (OR:6.35, 95\%CI: 1.28-31.56) of having adequate/controlled fasting glycaemia at final evaluation. PCS-diet at final evaluation was independently predicted by baseline PCS-diet $(\mathrm{B}=.31$, 95\%CI: .06-.64), variation in autonomous regulation $(\mathrm{B}=.43,95 \% \mathrm{CI}: .15-.71)$ and allocation group $(\mathrm{B}=.26$, 95\%CI: .04-1.36).

Conclusions: Results on weight and metabolic control support INDIVIDUO as a valuable clinical tool for obesity surgery candidates counseling. Additionally, intervention associated with perceived competence for weight-control behaviors and autonomous regulation.

\section{Keywords}

Bariatric surgery preoperative diet; Severe obesity; Patient centered care; Clinical effectiveness 


\section{Introduction}

The management of obesity, as a chronic and prevalent disease, represents an escalating challenge for healthcare systems. As it demands relevant patients' engagement in self-care, insufficient compliance with prescribed behaviors is frequently pointed-out as the main motive for diminished success of treatment[1]. Obesity-related social stigma, mainly focused on patients' weight, represents both a social justice issue and a public health challenge[2]. The excessive emphasis on patients' corpulence may determine treatment's expectancies to be focused on unreasonable weight reduction goals. For patients with severe obesity, the intensity and specificity of treatment needs to be in line with the magnitude of the disease, thus requiring differentiation and training for health professionals[3]. Obviously, when treating individuals suffering from severe obesity, it is difficult to diverge from weight loss as a primum movens for intervention. Nonetheless, the impact of the treatment on weight is only a piece, from a broader range of potential health gains, both at metabolic and health-related quality of life (HRQoL) levels.

The short- and medium-term impacts on weight loss and overall health improvements support the arguments for obesity surgery as the most effective treatment for extreme cases of obesity (class three or class two plus one or more comorbidity). Best practice guidelines [4-6] recommend the decision to adopt surgical treatment be contingent on a comprehensive interdisciplinary assessment, with the core team including specialists experienced in obesity management and bariatric surgery (namely, physician, surgeon, anesthetist, psychologist and/or psychiatrist, nutritionist or dietitian, nurse practitioner and social worker).

Nutritional counseling to obesity surgery candidates should aim at[4-6]: (a) contributing to the optimization of control of co-morbidities in order to reduce the risks associated with surgical procedures, (b) informing patients on the dietary changes that are required after surgery and (c) assessing patient motivation and willingness to adhere to post-operative behavior changes.

Exposure to highly palatable foods (e.g. those rich in sugar and fat) may challenge a patient's long-term commitment to a healthy diet. Increased food intake may be driven by the activation of brain reward-circuits, which result from the experience of pleasure coming from the consumption of such foods (hedonic-driven food intake)[7,8]. Nonetheless, individuals who maintain their weight (after intentionally losing it) report strategies like "finding the routine" and "being in control" as valuable for their success[9]. These strategies are in line with the relevance of self-perceived competence, at the core of the broader concept of selfdetermination as a key for adoption and maintenance of health-protecting behaviors[10-12]. Given the inherent complexity of behavior change, particularly food-related, nutritional intervention with obesity surgery candidates should aim at facilitating patients' engagement with lifestyle modification needed for successful (long-term) outcomes[5,6,13,14]. In broader terms, nutritional interventions should aim to contribute to patients' overall health improvements, benefiting those patients engaging in surgical treatment, as well as the ones who cannot or choose not to undergo surgical treatment.

Since severe obesity is a complex clinical condition, the nutritional intervention design should address multiple eating behaviors, be sustained by suitable theoretical frameworks and be delivered together with evidence-based health practices, namely behavior-change strategies[3,15]. While patient-centered nutritional counseling prior to bariatric surgery is generally considered a pre-requisite for assessment of obesity surgery candidates, we did not find any well-defined and evidence-based guidelines for this counseling. 
The therapeutic model INDIVIDUO (a Portuguese-derived acronym for "lifestyle-focused nutritional intervention in individuals with obesity") aims at fostering severely obese individuals with effective weightcontrol behaviors, by combining evidence-based nutritional strategies with a patient-centered counselling style.

The main goal of this study was to evaluate the effectiveness of INDIVIDUO as a therapeutic tool for obesity surgery candidates (i.e. in the context of pre-surgery obesity management). Specific goals are: (a) to evaluate the effect of INDIVIDUO in terms of weight control/reduction and metabolic improvement, namely, blood pressure (systolic and diastolic), fasting glycaemia, fasting insulin, hemoglobin A1c, fasting triglycerides, total cholesterol, LDL cholesterol and HDL cholesterol, and; (b) to evaluate the impact of INDIVIDUO in terms of eating patterns (reported number of meals) and light physical activity (in minutes), hedonic hunger, autonomous/controlled regulation for diet, perceived competence for diet (PCS-diet) and obesity-related quality of life.

\section{Materials and methods}

\section{Study design}

INDIVIDUO was tested with a prospective, randomized controlled single-site study, with two arms: intervention and control groups. Data were collected at baseline and at a 6-month re-assessment prior to consultations in a hospital setting. Questionnaires were self-administered, with the exception of variables related to socio-demographics, personal family history of obesity, comorbidities and pharmacotherapy use, and dietary and physical activity patterns, which were obtained through face-to-face interviews by trained professionals (not involved in the intervention).

\section{Target population and sampling}

The study included all adult patients referenced to the Obesity Treatment Unit of a Portuguese hospital within the six-months-period before the beginning of the fieldwork. Following national norms for obesity surgery[6], only patients with obesity Class III or Class II (in this case, with at least one comorbidity) are considered eligible for obesity surgery. Pregnant patients and/or patients with diagnosed mental impairment were excluded.

Eligible patients were stratified by sex and age groups (Younger: $<40$ years old; Middle-aged: 40-59 years; Older: > 59 years old), from which (subsample group) patients were randomly assigned to either the intervention or control group. The minimum sample size at the end of the trial was estimated to be of 25 patients per group. This was calculated taking into account the usual productivity of the clinic over a 6month period[16] (timeframe of the study) and considering an average of $27 \%$ no-shows to a first appointment and an attrition rate of $36 \%$. For minimizing inter-arm contamination, consultations with patients from the intervention group and from the control group were schedule in alternate weeks. From the census of patients $(n=147), 53$ did not participate (for reasons, see Figure 1). The total number of enrolled patients in the trial was 94 (45 in the intervention group and 49 in the control group). For gender and age distributions, no significant differences existed between participants and non-participants. 


\section{Characteristics of the intervention to be tested}

Format and frequency of appointments

To facilitate a more effective evaluation, usual appointment procedures of the outpatient clinic were reproduced. According to this, consultations were schedule every two months (i.e., four visits within the study timeframe), for the intervention group and twice for the control group (corresponding to baseline and final assessments).

\section{Counseling style and attitude}

INDIVIDUO centers itself around shared clinician-patient definition of goals and behavior change strategies, disregarding "behavioral prescription". Standing on the evidence that decision-making comes from an individual balance between the pros and cons of each behavior or goal[17,18], traditional directive prescription is replaced by the proposal of evidence-based strategies along with the invitation for patients to decide which strategies to adopt. Striving for a therapeutic setting which promotes shared-decisions, we have based our model on principles from the transtheoretical model of health behavior change[19], the selfdetermination theory[20] and motivational interviewing[17]. Four main techniques define the interaction with patients: asking open questions; expressing empathy; signaling discrepancy, and; contributing to enhanced self-efficacy. As an example, nutritionists may use an adapted version of the usual twenty-four hour food recall (or usual 24-hour food recall), based on: a) open question, to identify established habits/routines; b) active listening, enabling discussion about perceptions and beliefs on eating behavior, and; c) empathy for perceived constraints, regarding food access and choices (clinical, economic, cultural, etc.). Tailored dietary advice would: (a) promote awareness of discrepancies between eating behaviors and related values, (b) value the patients' past/present strategies that are convergent with health-gain needs, (c) contribute to the patients' perception of self-efficacy and (d) enhance change-talk[17,20].

\section{Nutritional counseling content}

In order to fully address patients' needs and therapy expectations, counseling ranges from the establishment of a full, personalized eating plan to a single strategy selection (see Figure 2 for strategies). INDIVIDUO uses three key principles for nutritional strategy selection and goal setting: a) basic nutritional needs satisfaction; b) therapeutic (weight and metabolic control) needs, and; c) adequacy to individuals' daily routines and constraints (personal agenda and values, financial limitations, etc.).

Weight loss results from a negative energy balance and, consequently, is contingent on patients' engagement with a reduced-calorie diet[21]. Nonetheless, estimating individual energy needs through mathematical formulae is susceptible to both over- and under-estimation.[22-25]. This risk is even higher when dealing with individuals with severe obesity (e.g., due to weight loss attempts' effects on body composition and metabolic rate) and it may represent an obstacle to adherence to dietary prescription and its clinical outcomes[26]. As a starting point, a reference-value of $1800 \mathrm{kcal} /$ day was adopted, based on four premises: a) it was proposed in weight-loss guidelines and other randomized trials[27,28]; b) it represents a 
light/moderate restriction to average expected individual needs[29,30]; c) it permits the inclusion of major food groups proposed by national guidelines [29]; d) it has been reported as the average energy consumption among successful weight losers[31].The characteristics of the baseline recommendations and the rational for the above-mentioned strategies were fully described elsewhere[32]. Since personalized guidance is the main strategy of INDIVIDUO, the referred baseline recommendations were used as a starting-point from which the nutritionist further developed an individualized eating plan for those in the intervention group. Energy and nutrient requirements were considered, as well as the selection of foods to provide them, taking into consideration patients' clinical condition, access, preferences and expectancies.

\section{Interventionists training and quality control of the intervention}

Before starting the study, two clinical nutritionists received three days (24-hours) of training, covering INDIVIDUO's nutritional and patient-centered strategies, with the principal researchers (JC and OS). Taped role-plays and in depth discussion took place during this training. Additionally, these nutritionists assisted 12 hours of consultations administered by JC (which strictly follows the INDIVIDUO principles). A standard operating procedure manual was created for the intervention. Across the trial, cases were discussed in weekly supervision meetings with JC and OS and other members of the research team.

\section{Control group intervention}

With regards to nutritional counseling, the control-group intervention was restricted to a literacy-promoting intervention, within the context of a formal appointment with a nutritionist (without training on the INDIVIDUO principles). This health-education intervention was based on the delivery and discussion of two documents: a leaflet with a list of weight-reduction strategies (similar to the ones proposed for the intervention group) and another leaflet describing a standard hypocaloric dietary plan, providing about 1800 $\mathrm{kcal} /$ day divided across six meals (similar to baseline recommendations used for intervention group, without personalization of the meal plan). The main characteristics of the interaction with controls took into consideration the national guidelines[6], which establish that multidisciplinary evaluation prior to obesity surgery must include nutritional guidance about healthy dietary habits and their modifications after surgery. Accordingly, participants of the control group were also informed about the typical diet after obesity surgery.

\section{Hypothesis to be tested}

The present study using INDIVIDUO is targeted to individuals with severe obesity, candidates to bariatric surgery. It uses a low-intensity[27] individual intervention (i.e., less-than-monthly treatment sessions), over a six-month period. We hypothesized that intervention would translate into: a) higher weight loss/control; b) better metabolic control; c) better patients' engagement, perceived competence and autonomous control for health promoting behaviors, and; d) positive impact on health-related quality of life (HRQoL).

\section{Variables, main outcomes and statistical analysis}

Socio-demographic (gender, age, literacy and professional situation), as well as personal and family history of obesity were assessed. Additionally, patients were asked about comorbidities (diabetes, arterial hypertension, dyslipidemia, osteoarticular disease) and pharmacotherapy use. Weight and height were 
measured by trained interviewers, using calibrated devices, according to official guidelines[33]. Body mass index (BMI) was computed as weight in kilograms divided by height in meters squared. Excessive weight was computed assuming desirable weight as the one corresponding to $\mathrm{BMI}=25$. Systolic blood pressure (SBP) and diastolic blood pressure (DBP) were measured according to national guidelines[34]. Blood samples, for fasting glycaemia, fasting insulin, glycated hemoglobin (HbA1c), fasting triglycerides, total cholesterol, LDL cholesterol (LDLc), and HDL cholesterol (HDLc) were collected after overnight fasting. Updated homeostatic model assessments (HOMA2), HOMA-\%S and HOMA-\%B were calculated and used as measures of beta cell function and insulin resistance[35]. Adequate glycemic control at final evaluation was assumed if [36]: a) fasting glycaemia was below $100 \mathrm{mg} / \mathrm{dL}$ in both evaluations; b) baseline fasting glycaemia was between 100 and $125 \mathrm{mg} / \mathrm{dL}$ and final (retest) fasting glycaemia was less than or equal to the baseline value or; c) baseline fasting glycaemia $>125 \mathrm{mg} / \mathrm{dL}$ and final (retest) fasting glycaemia was between 70 and $130 \mathrm{mg} / \mathrm{dL}$. The index TG/HDLc was computed and the cutoffs of 3.5 for men and 2.5 for women were used to identify individuals with insulin resistance and increased cardio-metabolic risk[37,38]. To get a general characterization of patient's habits and eating patterns, an adapted version of a twenty-four hour recall was applied at baseline and final evaluation. Obesity-specific Physical Activity Recall (ObPAR) $[39,40]$ was used to assess time spent in sedentary behavior and in light physical activity[41]. Patients perceived competence for dieting was assessed using the Portuguese version of the Perceived Competence Scale (PCS)[42]. Self-regulation was evaluated using a modified version of the Treatment Self-Regulation Questionnaire (TSRQ)[10,11]. The applied version was composed of 12 items (coefficient alpha: .819); ranging from .785 to .815 , when single items deleted), eight of them measuring autonomous regulation (coefficient alpha: .860) and four measuring controlled regulation (coefficient alpha: .732). Hedonic hunger (i.e., the drive to consume food in order to obtain pleasure) was assed using the Portuguese version of the questionnaire The Power of Food Scale (PFS)[8,43]. ORWELL-R was used to assess health-related quality of life[44].

Outcomes included weight, metabolic variables (blood pressure, glycaemia, insulinemia, triglycerides, and cholesterol), measures of eating and physical activity patterns, as well as hedonic hunger, autonomous/controlled regulation for diet, perceived competence for diet (PCS-diet) and quality of life. Primary outcomes were weight and metabolic control indicators. SPSS version 22.0 was used for statistical analyses. In line with international recommendations two distinct strategies of data analysis were done[45]: (1) intention-to-treat (ITT), as a primary analysis, and; (2) per-protocol, as a supportive analysis. The ITT analysis (treatment as assigned approach), considered all patients randomly allocated to both arms of the study at baseline with a last observation carried forward imputation for those who did not undergo the final evaluation. The per-protocol analysis (treatment as received approach), included only the subset of the ITT sample who completed both evaluations (baseline and final) without any major protocol violations. When describing differences between intervention and control group, results from both types of analysis are reported; for effect size assessment, only per-protocol approach results are reported in order to have an estimation of the impact of the treatment as received. The level of significance was always $95 \%$. Normality of distributions was assessed through Shapiro-Wilk test, as well as per analysis of kurtosis and skewness (values in the -2 through +2 range). Variation between baseline and final assessments were used for comparison between the two arms of the trial, controlling for confounding variables (see Results section for 
specific confounders for each variable). T-test or Mann-Whitney U test were used, depending on normality or non-normality, respectively. Linear and binary logistic regression (enter method) were used for assessing the effect size of the intervention (with odds ratio and $95 \%$ confidence intervals - 95\%CI). Cohens'd coefficient was used for estimation of effect sizes. Spearman's correlation was used to study associations between clinical, behavioral and HRQoL variables.

\section{Ethical issues}

The study follows the Code of Ethics of the Declaration of Helsinki[46]. The study protocol was approved by the Ethics Commission of the Faculty of Medicine of Lisbon and by the Clinical Direction of the Hospital de Santa Maria. Patients voluntarily agreed to participate in the study and signed a detailed informed consent document before engaging in the study. No incentives were provided for participation. Patients were informed that their participation would not imply any benefit or prejudice regarding the usual treatment process. Having this in mind, dropout was assumed when patients specifically declined further follow-up or when they missed two consecutive appointments without any justification. As previously indicated, usual waiting list time for treatment start-up was unaffected, either for the intervention group or for the control group patients (who were enrolled in regular treatment at the expected timing, according to their position in the waiting list).

\section{Results}

\section{Baseline characteristics and dropout rate}

In total, 94 patients participated in the trial: 45 for the intervention group and 49 for the control group. The baseline characteristics of the two groups were similar (Table 1).

Overall, 60 patients completed the trial, 29 of which completed the intervention program and participated in the final assessment (dropout rate: 35.5\%). From the 16 patients who did not finish the INDIVIDUO, 12 were women (four men), with a mean age of $43.19 \pm 12.73$ years old and a mean BMI of $42.41 \pm 5.80 \mathrm{~kg} / \mathrm{m}^{2}$. Most of them (nine patients) dropped out after the first visit, four after the second and one after the third. For the control group, 31 individuals participated in the final assessment; among patients who dropped-out $(38.0 \%) 14$ were women (four men), with a mean age of $47.00 \pm 13.64$ years old and a BMI of $41.83 \pm 5.78$ $\mathrm{kg} / \mathrm{m}^{2}$. No statistically significant differences were found between completers and non-completers for these variables (age and $\mathrm{BMI})$.

Most individuals reported that their obesity condition started as adults $(61.3 \%, \mathrm{n}=57)$, even though more than one fifth $(22.6 \%, \mathrm{n}=21)$ reported to suffer from obesity since birth. More than eighty percent $(81.9 \%, \mathrm{n}=77)$ reported to have someone also suffering from obesity in their close social network (family and/or friends) and more than three quarters $(75.5 \% ; n=71)$ reported a family history of obesity (i.e. presence of obesity in parents, grandparents and/or siblings). More than one-half $(52.1 \%, \mathrm{n}=49)$ of the individuals reported to have made ten or more previous attempts at weight loss. About forty percent of the individuals $(42.6 \% ; n=40 ;)$ were employed at the start of the study, with a similar percentage for the individuals completing (36.7\%; $\mathrm{n}=22 ;$ ) the six months assessment. 


\section{Impact on weight and metabolic control}

The impact on weight was significantly more pronounced in the intervention group (the following variables are presented as averages for intervention vs. control groups): body weight $(-4.21 \mathrm{~kg} v s .-0.21 \mathrm{~kg}, \mathrm{P}<0.01)$, BMI $\left(-1.69 \mathrm{~kg} / \mathrm{m}^{2}\right.$ vs. $\left.-0.11 \mathrm{~kg} / \mathrm{m}^{2}, \mathrm{P}<0.01\right)$; percentage weight loss $(-3.92 \%$ vs. $-0.27 \%, \mathrm{P}<0.01)$ and percentage of excessive weight loss (\%EWL: $9.68 \%$ vs. $0.51 \%, \mathrm{P}<0.01)$. When adjusting for age and baseline body mass index, the allocation group remains an independent predictor of excessive weight loss $\left(r^{2}=.18 ;\right.$ Adjusted $\left.r^{2}=.14 ; \mathrm{B}=8.43,95 \% \mathrm{CI}: 2.79-14.06, \mathrm{P}=0.01\right)$.

The decrease in body mass index had a positive association with improvement in fasting insulin $\left(\mathrm{r}_{\mathrm{s}}=0.443\right.$, $\mathrm{P}=0.02)$ and $\mathrm{HbA1c}\left(\mathrm{r}_{\mathrm{s}}=0.444, \mathrm{P}=0.02\right)$, but only for the intervention group. An overall positive impact from the intervention was observed for fasting glycaemia, insulin and glycated hemoglobin (Table 2). After excluding patients who reported intensification of diabetes-specific medication, significant impact from intervention on fasting glycaemia was detected (average variation: $-4.43 \mathrm{mg} / \mathrm{dL}$ for the intervention group vs. $+0.04 \mathrm{mg} / \mathrm{dL}$ for the control group; $\mathrm{P}=0.03$ ).

\section{INSERT TABLE 2 HERE}

A logistic regression, adjusting for age, baseline fasting glycaemia and \%EWL, modeled the impact of the intervention in achieving controlled fasting glycaemia at retest. The full model, containing all predictors, is statistically significant $\left(\chi^{2}(4, \mathrm{n}=56)=22.42, \mathrm{P}<0.01\right)$, explaining between $31.6($ Cox and Snell $R$ square $)$ and $43.8 \%$ (Nagelkerke $R$ squared) of the variance in glycemic control status (and able to correctly classify $83.1 \%$ of cases). This arm of the study independently contributed to glycemic control (OR=6.46 (95\%CI: 1.28-31.56); $\mathrm{P}=0.03)$ : patients who received intervention were over 6 times more likely to have contolled glycemic levels at retest (Table 3).

-INSERT TABLE 3 HERE

Both at baseline and final evaluations (Figure 3), controls had a significantly higher HOMA2 index and lower insulin sensitivity (HOMA-\%S). Concerning beta cell function (HOMA-\%S), groups differed significantly at final evaluation. For the intervention group, a decrease in HOMA2 and a significant improvement in HOMA-\%S were noticeable. Mean variations in insulin sensitivity were significantly different between groups $\left(t_{(51)}=-2.12 ; \mathrm{P}=0.04\right.$, effect size (Cohen's $\left.d\right)=0,61$ ).

INSERT FIGURE 3 HERE-

A multiple linear regression was run to predict insulin sensitivity improvement, with baseline insulin sensitivity (HOMA-\%S), percent weight variation and allocation group as the independent variables. All three variables were confirmed as predictors for insulin sensitivity improvement: baseline HOMA-\%S (B= $.57 ; 95 \% \mathrm{CI}:-.84$ to $-.30, \mathrm{P}<0.001), \% \mathrm{EWL}(\mathrm{B}=1.37,95 \% \mathrm{CI}: .65$ to $2.09, \mathrm{P}<0.001)$ and intervention 
( $\mathrm{B}=18.96,95 \% \mathrm{CI}: 4.30$ to 33.61, $\mathrm{P}=0.01)$. Overall model fit: $\mathrm{Z}_{(3,48)}=10,68, \mathrm{P}<0.001, \mathrm{r}^{2}=.40$; Adjusted $\mathrm{r}^{2}$ $=.36$.

At baseline, HOMA2 index was correlated with the TG/HDLc index $(\mathrm{r}=.307, \mathrm{P}<0.001)$. Using the same index (cutoffs: TG/HDLc $\geq 3.5$ for men and TG/HDLc $\geq 2.5$ for women), 38.4\% of the initial sample could be classified as having increased cardiovascular risk. For the intervention group, final TG/HDLc correlated inversely with weight loss $(\mathrm{r}=-.376 ; \mathrm{P}=0.04)$.

As shown in Table 2, lipid profile as well as blood pressure variations (between baseline and final observation) are not significantly different between groups.

\section{Impact on health-related quality of life, hedonic hunger, perceived competence and behavior change}

Although differences were not statistically significant, the intervention group showed a slightly higher average improvement in overall health-related quality of life (ORWELL-R total score: -11.04 vs. -6.58 for the control group), Body-environment experience subscale (-4.62 for intervention group vs. -3.38 for controls) and Illness perception \& distress subscale (-5.66 for intervention group vs. -0.55 for controls). For the subscale Physical symptoms, opposite results were encountered with a higher improvement in the control group (-2.65) than in the intervention group (-0.76). For the control group, HRQoL improvement and \%EWL were inversely correlated $(\mathrm{r}=-.38, \mathrm{P}=0.04)$. Percentage excessive weight loss was also negatively associated with test-retest decrease in hedonic hunger $(\mathrm{r}=-.37, \mathrm{P}=0.04)$. For the intervention group, reduction in hedonic hunger was positively correlated $(\mathrm{r}=.46, \mathrm{P}=0.01)$ with increments in perceived competence for diet (PCS-diet).

Baseline PCS-diet was negatively associated with the presence of obesity in an individual's close social network (partial correlation controlling for allocation group: $\mathrm{r}=-.24 ; \mathrm{P}=0.02$ ) and positively associated with both autonomous (partial correlation controlling for allocation group: $\mathrm{r}=.34 ; \mathrm{P}<0.01$ ) and controlled regulation (partial correlation controlling for allocation group: $\mathrm{r}=.27 ; \mathrm{P}<0.01$ ). Nonetheless, at final evaluation, only increases in autonomous regulation were associated with increases in PCS-diet (partial correlation controlling for allocation group: $\mathrm{r}=.52 ; \mathrm{P}<0.001)$. In the linear model $\left(\mathrm{Z}_{(4,53)}=5,01, \mathrm{p}<.001\right.$, $\mathrm{r}^{2}=.28$; Adjusted $\left.\mathrm{r}^{2}=.22\right)$, baseline PCS-diet $(\mathrm{B}=.31,95 \% \mathrm{CI}$ : .06 to $.64, \mathrm{P}=0.02)$, variation in autonomous regulation $(\mathrm{B}=.43,95 \% \mathrm{CI}$ : .15 to $.71, \mathrm{P}<0.001)$ and allocation group $(\mathrm{B}=.26,95 \% \mathrm{CI}$ : .04 to $1.36, \mathrm{P}=0.04)$ independently predicted final PCS-diet, whereas variation in controlled regulation were not associated with final PCS-diet. Considering the overall sample, enhancements in PCS-diet correlated positively with BMI reduction ( $\mathrm{r}=.27, \mathrm{P}=0.04)$ and with increases in reported number of meals $(\mathrm{r}=.32, \mathrm{P}=0.01)$. Those patients who reported similar or increased number of meals had a significantly higher $\%$ EWL $(6.77 \%$ vs. $1.29 \%$ for those patients reporting less meals at retest, compared to initial evaluation, $\mathrm{P}=0.03$ ). Also noteworthy is the observation that overall variation in the number of meals associated positively with the reported time (number of minutes) of light physical activity at retest ( $\mathrm{r}=.28, \mathrm{P}=0.03$ ).

\section{Discussion}

To our knowledge, this is the first study testing a low-intensity, patient-centered nutritional intervention, specifically tailored for individuals with severe obesity, namely obesity surgery candidates. Concerning the low-intensity characteristic, the frequency of contacts between patients and health-professionals may be (by 
itself) a determinant for the impact of the diet therapy and it has been suggested that comprehensive lifestyle interventions, which provide less-than-monthly treatment sessions, do not consistently result in weight loss when compared with usual care[27]. In these terms, it is relevant to stress that INDIVIDUO replicates the usual frequency of nutrition appointments in the Obesity Treatment Unit, which, due to operational issues, averages one appointment every two months. This allowed us to evaluate its impact on weight-loss (as compared to one nutritional-counseling and literacy-promotion session) in a real-life setting, assuring the ecological validity of the effectiveness assessment.

Despite the tendency of the intention-to-treat (ITT) analysis to dilute the treatment difference of interest[45], significant changes (test-retest differences) were observed for two primary outcomes (weight and fasting glycaemia) using both the ITT and the per-control analysis. Our results sustain INDIVIDUO' superiority compared to standard weight-control literacy promotion based counseling. Furthermore, since the main research goal for this study was to evaluate the effectiveness of a new intervention protocol (INDIVIDUO), the use of a per-protocol (non-intention to treat) analysis becomes adequate and its results more relevant. Concerning the relatively high dropout rate, it was similar to the Hospital's Obesity Treatment Unit's usual rate[16]. Therefore, being involved in this clinical trial was not a major contributor to patient dropout. Participants were mostly women, making it difficult to determine sex-specific responses. This overrepresentation of women replicates the usual gender distribution of the Unit and is comparable to other obesity surgery studies[47,48]. The six-month length of the study disallows inference about long-term impact of INDIVIDUO. Nonetheless, other studies adopted similar, or even shorter, intervention periods[48-50].

Additionally, the six-month duration of the intervention enabled individuals allocated to the control group to initiate regular treatment at the expected timing, according to their position in the waiting list. Therefore, they were not affected in terms of their normal treatment process.

Thought not consensual, weight loss prior to obesity surgery may be required, based on clinical and reimbursement contingencies, such as the ones demanded by insurance companies. Still et al[48] showed that obesity surgery candidates who achieved a 5\% to $10 \%$ decrease in excessive body weight before surgery lost weight more rapidly and had a higher probability of a shorter hospital stay postoperatively. Benotti et al[50] used a 6-month multidisciplinary program that encouraged candidates to lose $10 \%$ of excessive weight prior to surgery and concluded that preoperative weight loss is associated with fewer complications after gastric bypass surgery. Going back to the work from Still et al[48], beginning with an approximate 500-kcal deficit and a macronutrient composition of a prudent low-fat diet, patients were instructed to follow a 1000- to 1500-kcal liquid diet if they had not reached their weight loss goal by month 4 . In our study, for individuals in the intervention group, the average excessive weight loss was just below $10 \%$, with nearly seventy percent losing $5 \%$ or more. These numbers are similar to the ones reported by Still et al[48]. We should highlight that weight loss goals were not pre-established for the intervention group (the eligibility criterion for the Unit does not include such a premise).

Although using meal replacements (liquid and/or prepackaged meal replacements) may be a valid strategy in obesity management[51], we are convinced that one strong point in favor of INDIVIDUO is the fact that patients in the intervention group achieved clinically relevant weight loss using conventional foods. The effectiveness of the adopted patient-centered nutritional counseling strategy (ranging from the definition of a full, personalized eating plan to a single strategy selection) is reinforced by the significant impact on glucose 
homeostasis. Our results are in line with the ones coming from the Finnish Diabetes Prevention Study[52], which used individually tailored dietary advice, regular foods and a frequency of contacts (between patients and nutritionists) similar to the ones chosen for INDIVIDUO. Our results for average decrease in fasting glycaemia for the treated individuals $(-4.43 \mathrm{mg} / \mathrm{dl})$ are, in fact, quite similar to the ones reported by Tuomilehto and colleagues $(-4 \mathrm{mg} / \mathrm{dl})[52]$. Both, their results and ours, on fasting insulin failed to show statistically significant results. Nonetheless, they have shown significant differences after oral glucose challenge[52]. We have not performed a glucose tolerance test. Instead, we used HOMA2, HOMA-\%S and HOMA- $\% B$, as surrogate indexes for insulin sensitivity/resistance. Therefore, our data does not permit any specific conclusions on stimulated glucose and insulin systems (i.e. peripheral glucose uptake)[53]. Nonetheless, we found significant decreases in fasting glycaemia and a six-fold probability of having it controlled, significant decreases in insulin resistance and decreases in beta cell function (significantly lower final HOMA-\%B) in the intervention group. These data are suggestive of an overall impact on glucose homeostasis.

It was our expectation that the intervention would have a positive impact on lipid profile. The apparent absence of impact may be contingent on baseline average values (for triglycerides, total cholesterol, LDLc and HDLc), within normal ranges. Therefore, the non-significant variations may only mean that individuals maintained a desirable lipid profile. Within this hypothesis, it becomes noticeable that a very relevant proportion of those individuals receiving the intervention had adequate LDLc and HDLc. The proportion of patients achieving adequate blood pressure values was just above $25 \%$, for both groups. Nonetheless, and without significant differences between groups, test-retest decreases for both SBP and DBP were observed. The decreases on SBP were less than the ones obtained with the low-sodium phase of the DASH diet (-8.9 $\mathrm{mmHg}$, as compared to the high-sodium phase of the control diet), but quite similar to the ones detected on DBP (-4.5 mmHg, for the same comparison)[54].

In their work, Benotti et al[50] acknowledge that preoperative weight loss alone may be a weak predictor of outcomes, and they propose other measures such as motivation, compliance, intellectual capability, social support, and economic status. According to these authors, such psychosocial variables can explain weight loss and moderate post-surgery results[50]. Our results also challenge the valuation of weight loss by itself. In fact, for the control group, weight loss was associated with decreases in HRQoL and increases in hedonic hunger. In the opposite direction, for the intervention group, the decrease in hedonic hunger was associated with increases in perceived confidence for diet.

The baseline inverse association between PCS-diet and the presence of obesity in a patients' close social network can be supported by data from Christakis and Fowler[55], specifically the psychosocial shaping of an individual's norms about overweightness and behaviors (e.g., food consumption patterns, physical activity engagement, etc.). Since a high proportion of our patients had close and regular contact with other persons with obesity, long-duration obesity and several failed attempts to lose weight, it becomes understandable that baseline PCS-diet associates not only with autonomous, but also controlled regulation. Within this context, Harrys and Hagger[56] noted that dieting tends to be a social behavior, with individuals pondering their own needs along with the opinions of others, when deciding to engage in dieting behavior. A strong point in favor of INDIVIDUO is that final PCS-diet is predicted by the intervention as well as by increments in autonomous regulation, without a significant predictive impact of controlled regulation. These results are 
similar to those presented by other authors, who suggest that long-lasting behavior change becomes more probable if individuals endorse weight loss-related behavioral goals through feeling competent and autonomous about reaching them[57]. Our data also suggest that enhancements in PCS-diet translate into weight loss and a change in food-intake pattern (i.e. increase in reported number of meals). The first association can be read backwards, i.e., weight loss improves patient's perceived competence for diet. Nonetheless, since general and food-specific (e.g., palatable foods) underreporting is prevalent among subjects with obesity[58], the association between enhancements in PCS-diet and reported number of meals is more in favor of perceived competence being related to higher awareness and engagement with behavior change. This is reinforced by the positive association between reported eating episodes and amount of light physical activity and by the fact that maintaining or increasing meals were associated with larger amounts of excessive weight loss.

Given its wide prevalence and comorbid conditions, obesity is a prominent problem for all health actors. In this paper, we describe and report findings on the effectiveness of INDIVIDUO. This intervention, directed to candidates for obesity surgery, integrates evidenced-based weight control strategies with a patientcentered clinical approach. Without disregarding the clinical relevance of the individuals' excessive weight, obesity-dedicated health professionals must challenge the paradigm of weight loss as their clinical "core business". Better said, when approaching individuals with severe obesity, the challenge may not be to promote weight reduction by itself, but rather to help them achieve health gains that ultimately translate into long-term weight control. Considering the presented data, INDIVIDUO is a promising tool for pre-surgery nutritional counseling, deserving further research. In the challenging field of obesity management, next steps for INDIVIDUO would be to clarify its impact on medium/long-term surgery outcomes, specifically, on patients' self-care competencies regarding lifestyle and, ultimately, weight, comorbidities and overall sustained gains in obesity-related quality of life.

\section{Competing interests}

The authors declare that they have no competing interests.

\section{Author's contributions}

JC and OS designed the study, performed the statistical analysis and drafted the manuscript. All authors critically reviewed the manuscript and approved the final version.

\section{Acknowledgements}

Authors would like to thank Alexandra Dinis for her valuable inputs regarding the statistical analysis. This project would not be possible without the diligent and enthusiastic participation of all remaining research team members: Dr. Luciana Cunha and Dr. Patrícia Rama, who conducted the consultations within the intervention arm; Dr. Rui Varela, who conducted the consultations within the control arm; Dr. Nuno Santos, Dr. Inês Soares, Dr. Joana Vitorino and Dr. Liliana Falcato as interviewers for data collection; Dr. João Martins, Dra. Inês Soares and Dra. Joana Vitorino for data insertion. Likewise, the project would be unfeasible without Prof. Mário Mascarenhas (Serviço de Endocrinologia do Hospital de Santa Maria, CHLN) and Prof. Fátima Reis (Instituto de Saúde Ambiental - ISAMB) willingness to endorse it as a relevant 
project, assuring necessary logistic support. The final version of this paper highly benefited from the valuable edition and text-revision from Hannah Frawley Freire. Finally, a word of gratitude to each patient who accepted to take part in this study.

\section{References}

[1] Abete I, Parra MD, Zulet M a, Martínez J a. Different dietary strategies for weight loss in obesity: role of energy and macronutrient content. Nutr Res Rev 2006;19:5-17. doi:10.1079/NRR2006112.

[2] Puhl RM, Heuer C a. Obesity stigma: important considerations for public health. Am J Public Health 2010;100:1019-28. doi:10.2105/AJPH.2009.159491.

[3] Dietz WH, Baur LA, Hall K, Puhl RM, Taveras EM, Uauy R, et al. Management of obesity: improvement of health-care training and systems for prevention and care. Lancet 2015;6736:1-13. doi:10.1016/S0140-6736(14)61748-7.

[4] Mechanick JI, Youdim A, Jones DB, Timothy Garvey W, Hurley DL, Molly McMahon M, et al. Clinical Practice Guidelines for the Perioperative Nutritional, Metabolic, and Nonsurgical Support of the Bariatric Surgery Patient-2013 Update: Cosponsored by American Association of Clinical Endocrinologists, The Obesity Society, and American Society fo. Surg Obes Relat Dis 2013;9:159_ 91. doi:10.1016/j.soard.2012.12.010.

[5] Fried M, Yumuk V, Oppert J-M, Scopinaro N, Torres AJ, Weiner R, et al. Interdisciplinary European Guidelines on Metabolic and Bariatric Surgery. Obes Facts 2013;6:449-68. doi:10.1159/000355480.

[6] Direcção-Geral da Saúde. Boas práticas na abordagem do doente com obesidade elegível para cirurgia bariátrica. vol. 2. 2012.

[7] Yu Y-H, Vasselli JR, Zhang Y, Mechanick JI, Korner J, Peterli R. Metabolic vs. hedonic obesity: a conceptual distinction and its clinical implications. Obes Rev 2015:1-14. doi:10.1111/obr.12246.

[8] Ribeiro G, Santos O, Camacho M, Torres S, Mucha-vieira F, Sampaio D, et al. Translation, Cultural Adaptation and Validation of the Power of Food Scale for Use by Adult Populations in Portugal $2015 ; 28: 575-82$.

[9] Lindvall K, Larsson C, Weinehall L, Emmelin M. Weight maintenance as a tight rope walk - a Grounded Theory study. BMC Public Health 2010;10:51. doi:10.1186/1471-2458-10-51.

[10] Ryan RM, Connell JP. Perceived locus of causality and internalization: Examining reasons for acting in two domains. J Pers Soc Psychol 1989;57:749-61. doi:10.1037/0022-3514.57.5.749.

[11] Williams GC, Minicucci DS, Kouides RW, Levesque CS, Chirkov VI, Ryan RM, et al. Selfdetermination, smoking, diet and health. Health Educ Res 2002;17:512-21.

[12] Teixeira PJ, Carraça E V., Marques MM, Rutter H, Oppert J-M, Bourdeaudhuij I De, et al. Successful behavior change in obesity interventions in adults: a systematic review of self-regulation mediators. BMC Med 2015;13:84. doi:10.1186/s12916-015-0323-6.

[13] Wansink B. From mindless eating to mindlessly eating better. Physiol Behav 2010;100:454-63. doi:10.1016/j.physbeh.2010.05.003.

[14] Engel G. The Need for a New Medical Model: A Challenge for Biomedicine. Science (80- ) 1977;196:129-236. doi:10.1126/science.847460.

[15] Mesas, AE; Muñoz-Pareja, M; López-García, E; Rodríguez-Artalejo F. Selected eating behaviours and excess body weight: a systematic review. Obes Rev 2012;13:106-35.

[16] Camolas J, do Carmo I, Moreira P, Santos O, Martins J, Jorge E, et al. Avaliação da Efectividade Terapêutica de uma Intervenção Nutricional em Indivíduos com Obesidade Grave. Rev Port Endocrinol Diabetes Metab 2014;8:118.

[17] Miller W, Rollnick S. Motivational interviewing: preparing people for change. 2nd ed. New York: The Guilford Press; 2002.

[18] Prochaska JO, Velicer WF, Rossi JS, Goldstein MG, Marcus BH, Rakowski W, et al. Stages of change and decisional balance for 12 problem behaviors. Heal Psychol 1994;13:39-46.

[19] Prochaska JO, DiClemente C, Norcross J. In search of how people change. Am Psychol 
1992;47:1102-14.

[20] Ryan R, Deci E. Self-determination theory and the facilitation of intrinsic motivation. Am Psychol 2000;55:68-78. doi:10.1037/0003-066X.55.1.68.

[21] Sacks F, Bray G, Carey V, Smith S, Ryan D, Anton S, et al. Comparison of Weight-Loss Diets with Different Compositions of Fat, Protein, and Carbohydrates. N Engl J Med 2009;360:859-73.

[22] Camolas J, Santos O, Moreira P, Do Carmo I. Promovendo mudanças comportamentais sustentáveis no controlo do peso corporal. Acta Med Port 2014;27:99-107.

[23] Henry C. Basal metabolic rate studies in humans: measurement and development of new equations.pdf. Public Health Nutr 2005;8:1133-52.

[24] Byrne NM, Wood RE, Schutz Y, Hills AP. Does metabolic compensation explain the majority of less-than-expected weight loss in obese adults during a short-term severe diet and exercise intervention? Int J Obes 2012;36:1472-8. doi:10.1038/ijo.2012.109.

[25] Schwartz A, Kuk J, Lamothe G, Doucet E. Greater than predicted decrease in resting energy expenditure and weight loss: results from a systematic review. Obesity 2012;20:2307-10.

[26] Blundell JE, Caudwell P, Gibbons C, Hopkins M, Naslund E, King N, et al. Role of resting metabolic rate and energy expenditure in hunger and appetite control: a new formulation. Dis Model Mech 2012;5:608-13. doi:10.1242/dmm.009837.

[27] Jensen MD, Ryan DH, Apovian CM, Ard JD, Comuzzie AG, Donato K a., et al. 2013 AHA/ACC/TOS guideline for the management of overweight and obesity in adults: A report of the American College of cardiology/American Heart Association task force on practice guidelines and the obesity society. Circulation 2014;129:S102-38. doi:10.1161/01.cir.0000437739.71477.ee.

[28] The Look AHEAD Research Group. The Look AHEAD study: a description of the lifestyle intervention and the evidence supporting it. Obesity (Silver Spring) 2006;14:737-52. doi:10.1038/oby.2006.84.

[29] Franchini B, Rodrigues S, Graça P, Vaz de Almeida M. A nova Roda dos Alimentos. . um guia para a escolha alimentar diária! Nutrícias 2004;4:55-6.

[30] Kee A, Isenring E, Hickman I, Vivanti A. Resting energy expenditure of morbidly obese patients using indirect calorimetry: a systematicreview. Obes Rev 2012;13:753-65.

[31] Wing RR, Phelan S. Long-term weight loss maintenance. Am J Clin Nutr 2005;82:222S - 225S.

[32] Camolas J, dos Santos O, Mascarenhas M, Moreira P, do Carmo I. INDIVIDUO: intervenção nutricional direcionada aos estilos de vida em indivíduos com obesidade. Acta Port Nutr 2015;3:1421. doi:10.21011/apn.2015.0303.

[33] Direcção Geral da Saúde. Orientação Avaliação Antropométrica no Adulto. Lisboa: 2013.

[34] Saúde D-G da. Hipertensão Arterial: definição e classificação. Direção-Geral Da Saúde 2013:1-6. file://C:/Users/User/Downloads/i018827.pdf.

[35] Wallace TM, Levy JC, Matthews DR. Use and abuse of HOMA modeling. Diabetes Care 2004;27:1487-95. doi:10.2337/diacare.27.6.1487.

[36] American Diabetes Association. Standards of Medical Care in Diabetes - 2015. Diabetes Care 2015;38. doi:10.2337/dc15-S005.

[37] McLaughlin T, Reaven G, Abbasi F, Lamendola C, Saad M, Waters D, et al. Is there a simple way to identify insulin-resistant individuals at increased risk of cardiovascular disease? Am J Cardiol 2005;96:399-404. doi:http://dx.doi.org/10.1016/j.amjcard.2005.03.085.

[38] Salazar MR, Carbajal H a., Espeche WG, Aizpurúa M, Leiva Sisnieguez CE, March CE, et al. Identifying cardiovascular disease risk and outcome: Use of the plasma triglyceride/high-density lipoprotein cholesterol concentration ratio versus metabolic syndrome criteria. J Intern Med 2013;273:595-601. doi:10.1111/joim.12036.

[39] Aadahl M, Jørgensen T. Validation of a new self-report instrument for measuring physical activity. Med Sci Sport Exerc 2003;35:1196-202.

[40] Castro M, Teixeira P, Vieira P, Silva M, Minderico C, Coutinho S, et al. Testing a new self-report instrument for measuring lifestyle physical activity in obese women. Int J Obes 2008;32:S176.

[41] Baptista F, Silva A, Santos D, Mota J, Santos R, Vale S, et al. Livro Verde da Actividade Física. 
Lisboa: 2011.

[42] Mestre S, Pais Ribeiro J. Adaptação de três questionários para a população portuguesa baseados na teoria de auto-determinação. In: Leal I, Pais-Ribeiro J, Silva I, Marques S, editors. Actas do $7^{\circ}$ Congr. Nac. Psicol. da saúde, Lisboa: ISPA; 2008, p. 623-6.

[43] Lowe M, Butryn M, Didie E, Annunziato R, Thomas J, Crerand C, et al. The Power of Food Scale. A new measure of the psychological influence of the food environment. Appetite 2009;53:114-8.

[44] Camolas J, Ferreira A, Mannucci E, Mascarenhas M, Carvalho M, Moreira P, et al. Assessing quality of life in severe obesity: development and psychometric properties of the ORWELL-R. Eat Weight Disord - Stud Anorexia, Bulim Obes 2015. doi:10.1007/s40519-015-0222-z.

[45] Gupta S. Intention-to-treat concept: A review. Perspect Clin Res 2011;2:109-12. doi:10.4103/22293485.83221 .

[46] World Medical Association. Ethical Principles for Medical Research Involving Human Subjects. Eur J Emerg Med 2008;8:221-3.

[47] Goodpaster BH, Delany JP, Otto AD, South-paul JE, Thomas SB, Brown J, et al. Effects of Diet and Physical Activity Interventions on Weight Loss and Cardiometabolic Risk Factors in Severely Obese Adults. JAMA 2010;304:1795-802.

[48] Still CD, Benotti P, Wood GC, Gerhard GS, Petrick A, Reed M, et al. Outcomes of preoperative weight loss in high-risk patients undergoing gastric bypass surgery. Arch Surg 2007;142:994-8; discussion 999. doi:10.1001/archsurg.142.10.994.

[49] Heinberg LJ, Schauer PR. Pilot testing of a portion-controlled, commercially available diet on presurgical weight loss and metabolic outcomes in patients undergoing bariatric surgery. Obes Surg 2014;24:1817-20. doi:10.1007/s11695-014-1371-2.

[50] Benotti P, Still C, Wood G, Akmal Y, King H, El Arousy H, et al. Preoperative Weight Loss Before Bariatric Surgery. Arch Surg 2009;144:1150-5. doi:10.1001/archsurg.2009.209.

[51] Keogh JB, Clifton PM. The role of meal replacements in obesity treatment. Obes Rev 2005;6:22934. doi:10.1111/j.1467-789X.2005.00171.x.

[52] Tuomilehto J, Lindstom J, Eriksson J, Valle T, Hämäläinen H, Ilanne-Parikka P, et al. Prevention of Type 2 Diabetes Mellitus by Changes in Lifestyle Among Subjects With Impaired Glucose Tolerance. N Engl J Med 2001;344:1343-50.

[53] Patarrão RS, Wayne W, Paula M. Assessment of methods and indexes of insulin sensitivity. Rev Port Endocrinol, Diabetes E Metab 2014;9:65-73.

[54] Sacks F, Svetkey L, Vollmer W, Appel L, Bray G, Harsha D, et al. Effects on Blood Pressure of Reduced Dietary Sodium and the Dietary Approaches to Stop Hypertension (DASH) Diet. N Engl J Med 2001;344:3-10.

[55] Christakis N a, Fowler JH. The spread of obesity in a large social network over 32 years. N Engl J Med 2007;357:370-9. doi:10.1056/NEJMsa066082.

[56] Harris J, Hagger M. Do Basic Psychological Needs Moderate Relationships Within the Theory of Planned Behavior? J Appl Biobehav Res 2007;12:43-64.

[57] Teixeira PJ, Silva MN, Mata J, Palmeira AL, Markland D. Motivation, self-determination, and longterm weight control. Int J Behav Nutr Phys Act 2012;9:22. doi:10.1186/1479-5868-9-22.

[58] Lissner L. Measuring food intake in studies of obesity. Public Health Nutr 2002;5:889-92. doi:10.1079/PHN2002388. 


\begin{tabular}{|c|c|}
\hline $\begin{array}{l}\text { Decrease } \\
\text { energy } \\
\text { density of } \\
\text { diet }\end{array}$ & $\begin{array}{l}\text { - Replace equivalent proportions of energy-dense and nutritionally poor foods by } \\
\text { nutritionally rich ones } \\
\text { - Diminish sugar intake by avoiding added sugars and sugar-sweetened beverages } \\
\text { - Control fat intake by avoiding high-fat foods and fat addition (as spread, } \\
\text { condiment and dressing) } \\
\text { - Increase water-rich vegetable proportions in meals: soups, garnish and recipe } \\
\text { (novel) ingredients } \\
\text { - Adopt Mediterranean cooking methods and ingredients with a twist: selecting } \\
\text { low-fat ingredients and trim fat, controlling added fat in sautéed preparations, and } \\
\text { using herbs and spices to reduce salt addition }\end{array}$ \\
\hline $\begin{array}{c}\text { Adopt } \\
\text { regular } \\
\text { eating } \\
\text { patterns }\end{array}$ & $\begin{array}{l}\text { - Engage in a stable day-to-day meal pattern of, at least, three meals } \\
\text { - Adopt breakfast, lunch and dinner as anchor meals } \\
\text { - Consider intermediary eating episodes (e.g., pre-planned snacks) if useful for } \\
\text { appetite control and for optimizing food selection } \\
\text { - Increase awareness on food selection and portion control, when meal patterns are } \\
\text { unstable or not possible }\end{array}$ \\
\hline $\begin{array}{c}\text { Decrease } \\
\text { eating rate }\end{array}$ & $\begin{array}{l}\text { - Diminish the size of each forkful, put down tableware between forkfuls or go } \\
\text { along with a slow-eating commensal }\end{array}$ \\
\hline $\begin{array}{l}\text { Engage } \\
\text { with } \\
\text { regular } \\
\text { physical } \\
\text { activity }\end{array}$ & $\begin{array}{l}\text { - Decrease time spend with sedentary behaviors (screen time, seated activities) } \\
\text { - Increase non-structured physical activities, such as walking to workplace/school, } \\
\text { using stairs (instead of elevators), engage in housekeeping and gardening activities, } \\
\text { dancing, etc. } \\
\text { - Engage in advised/accompanied structured physical exercise, whenever available } \\
\text { and if suitable for the patient }\end{array}$ \\
\hline
\end{tabular}

Figure 1: List of objectives and strategies for patients' guidance within INDIVIDUO. 


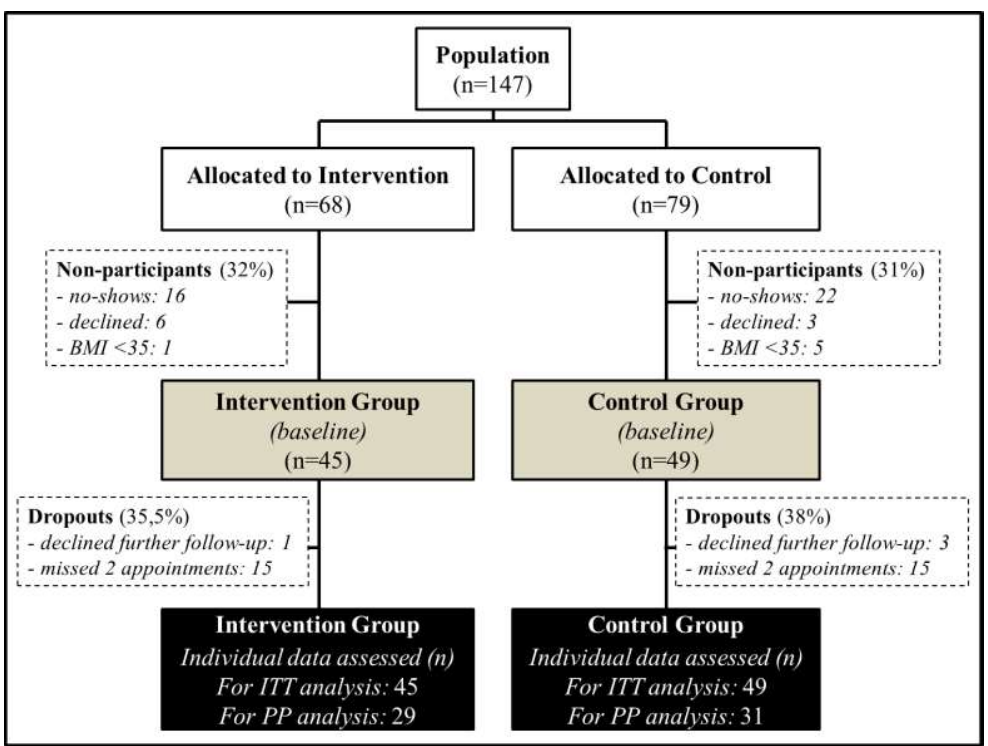

Figure 2. Allocation algorithm 


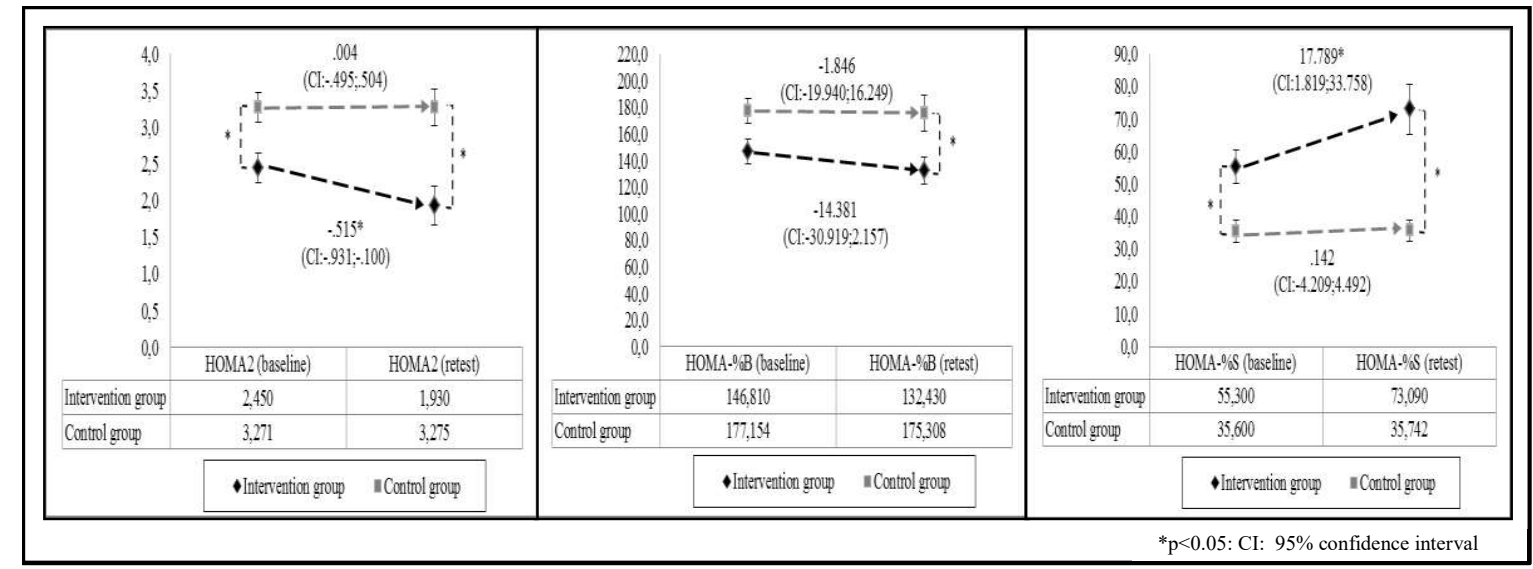

Figure 3: Homeostatic model assessment (HOMA2), insulin sensitivity (HOMA-\%S) and beta cell function (HOMA-\%B) variation, by group (per-protocol, corrected for medication intensification and for model specifications: 26 patients from intervention group and 24 from the control group were used in this analysis) 
Table 1.

Baseline characteristics of participants (intervention and control groups)

\begin{tabular}{|c|c|c|c|c|}
\hline \multicolumn{2}{|l|}{ Variable } & $\begin{array}{c}\text { Intervention group } \\
(\mathrm{n}=45)\end{array}$ & $\begin{array}{l}\text { Control group } \\
\qquad(\mathrm{n}=49)\end{array}$ & $\mathbf{P}^{*}$ \\
\hline \multicolumn{2}{|l|}{ Female: $n(\%)$} & $37(82.2)$ & $39(79.6)$ & ns \\
\hline \multirow{4}{*}{ Age } & Younger-ages $(<40$ years): $\mathrm{n}(\%)$ & $14(31.0)$ & $20(40.8)$ & \multirow{3}{*}{ ns } \\
\hline & Middle-aged (40-59 years): n (\%) & $23(51.0)$ & $23(46.9)$ & \\
\hline & Older-ages $(\geq 60$ years): $\mathrm{n}(\%)$ & $8(17.8)$ & $6(12.2)$ & \\
\hline & mean \pm standard deviation & $46.31 \pm 13.65$ & $43.53 \pm 13.92$ & ns \\
\hline \multicolumn{2}{|c|}{ Body-mass Index $^{\dagger}$} & $42.81 \pm 4.96$ & $43.45 \pm 7.04$ & ns \\
\hline \multirow{5}{*}{$\begin{array}{l}\text { Comorbidity } \\
\text { (self-report) }\end{array}$} & Any type: n (\%) & $33(73.3)$ & $35(71.4)$ & ns \\
\hline & Diabetes: $\mathrm{n}(\%)$ & $9(20.0)$ & $14(28.6)$ & ns \\
\hline & Dyslipidemia: n (\%) & $20(44.4)$ & $14(28.6)$ & ns \\
\hline & Hypertension: $\mathrm{n}(\%)$ & $26(57.8)$ & $24(49.0)$ & ns. \\
\hline & Osteoarticular disease: $\mathrm{n}(\%)$ & $6(13.3)$ & $8(16.3)$ & $\mathrm{ns}$ \\
\hline \multirow{3}{*}{$\begin{array}{l}\text { Medication } \\
\text { intake } \\
\text { (self-report) }\end{array}$} & Diabetes: $\mathrm{n}(\%)$ & $9(20.5)$ & $11(22.4)$ & ns \\
\hline & Dyslipidemia: n (\%) & $12(27.3)$ & $11(22.4)$ & ns \\
\hline & Hypertension: n (\%) & $17(38.6)$ & $21(42.9)$ & ns \\
\hline \multirow{3}{*}{$\begin{array}{l}\text { Glucose } \\
\text { homeostasis }\end{array}$} & Fasting glycaemia $(\mathrm{mg} / \mathrm{dl})$ & $96.44 \pm 14.36$ & $104.05 \pm 16.90$ & ns \\
\hline & Hemoglobin Alc $(\%)$ & $5.82 \pm 0.69$ & $5.84 \pm 0.67$ & ns \\
\hline & Fasting insulin $(\mathrm{mU} / \mathrm{L})$ & $21.21 \pm 14.60$ & $24.13 \pm 11.52$ & ns \\
\hline \multirow{2}{*}{ Blood pressure } & Systolic $(\mathrm{mmHg})$ & $142.78 \pm 19.29$ & $139.84 \pm 16.15$ & ns \\
\hline & Diastolic $(\mathrm{mmHg})$ & $84.64 \pm 11.11$ & $85.82 \pm 10.44$ & ns \\
\hline \multirow{4}{*}{ Lipid profile } & Fasting triglycerides $(\mathrm{mg} / \mathrm{dl})$ & $118.67 \pm 56.48$ & $126.14 \pm 50.08$ & ns \\
\hline & Total cholesterol (mg/dl) & $188,00 \pm 40.42$ & $188.21 \pm 33.13$ & ns \\
\hline & LDL cholesterol $(\mathrm{mg} / \mathrm{dl})$ & $111.70 \pm 34.38$ & $113.32 \pm 24.30$ & ns \\
\hline & HDL cholesterol (mg/dl) & $52.67 \pm 13.98$ & $50.0 \pm 11.47$ & ns \\
\hline \multirow{4}{*}{$\begin{array}{l}\text { Health-related } \\
\text { quality of life } \\
(O R W E L L-R)\end{array}$} & Overall score & $240.29 \pm 80.17$ & $234.96 \pm 101.15$ & ns \\
\hline & Body-environment experience & $126.89 \pm 45.12$ & $128.88 \pm 53.91$ & ns \\
\hline & Illness perception \& distress & $93.02 \pm 34.99$ & $82.98 \pm 37.74$ & ns \\
\hline & Physical symptoms & $20.38 \pm 14.51$ & $23.10 \pm 16.65$ & ns \\
\hline
\end{tabular}

${ }^{\ddagger} \mathrm{P}$-values are for the intervention-control inter-group comparisons; n.s. for non-significant differences, at $\alpha=.05$; ${ }^{\dagger} \mathrm{Body}-$ mass index: weight in kilograms divided by the square of the height in meters 


\section{Table 2.}

Variation (by group) in weight, glucose homeostasis, blood pressure and lipid profile ${ }^{*}$

\begin{tabular}{|c|c|c|c|c|c|c|}
\hline & \multicolumn{3}{|c|}{$\begin{array}{c}\text { Intention-to-treat analysis } \\
\text { (with last observation carried forward) }\end{array}$} & \multicolumn{3}{|c|}{$\begin{array}{c}\text { Per-protocol analysis } \\
\text { (patients who completed both evaluations) }\end{array}$} \\
\hline & $\begin{array}{c}\text { Intervention } \\
\text { group } \\
(n=45)\end{array}$ & $\begin{array}{c}\text { Control } \\
\text { group } \\
(n=49)\end{array}$ & P-value $^{\dagger}$ & $\begin{array}{c}\text { Intervention } \\
\text { group } \\
(n=29)\end{array}$ & $\begin{array}{l}\text { Control } \\
\text { group } \\
(n=31)\end{array}$ & P-value $^{\dagger}$ \\
\hline$B M I$ & $-1.09(1.53)$ & $-0.07(1.45)$ & .001 & $-1.69(1.62)$ & $-0.11(1.83)$ & .001 \\
\hline Weight lost (\%) & $-2.53(3.52)$ & $-0.17(3.33)$ & .001 & $-3.92(3.72)$ & $-0.27(4.21)$ & .001 \\
\hline Excessive weight lost (\%) & $-6.24(8.80)$ & $-0.32(9.07)$ & .001 & $-9.68(9.34)$ & $-0.51(11.47)$ & .001 \\
\hline$>5 \%$ excessive weight lost (\%) & 24,4 & 6,1 & \multirow{2}{*}{.008} & 37,9 & 9,7 & \multirow{2}{*}{.002} \\
\hline$>10 \%$ excessive weight lost $(\%)$ & 20,0 & 10,2 & & 31,0 & 16,1 & \\
\hline Fasting glycaemia $(\mathrm{mg} / \mathrm{dl})^{*}$ & $-1.91(6.72)$ & $1.24(8.65)$ & .019 & $-3.03(8.33)$ & $2.17(11.46)$ & .030 \\
\hline Hemoglobin Alc (\%) & $-0.13(0.33)$ & $-0.05(0.18)$ & n.s. & $-0.20(0.39)$ & $-0.09(0.23)$ & n.s. \\
\hline Fasting insulin $(m U / L){ }^{*}$ & $-1.71(8.27)$ & $0.05(7.02)$ & n.s. & $-2.73(10.37)$ & $0.09(9.37)$ & n.s. \\
\hline Fasting triglycerides $(\mathrm{mg} / \mathrm{dl})^{*}$ & $1.91(30.72)$ & $1.87(39.90)$ & n.s. & $3.04(39.00)$ & $3.04(51.11)$ & n.s. \\
\hline Total cholesterol $(\mathrm{mg} / \mathrm{dl})^{*}$ & $0.37(20.23)$ & $7.55(23.17)$ & n.s. & $0.59(25.71)$ & $1.21(29.68)$ & n.s. \\
\hline LDL cholesterol $(\mathrm{mg} / \mathrm{dl})^{*}$ & $1.63(20.41)$ & $2.68(19.78)$ & n.s. & $2.59(25.89)$ & $4.35(25.21)$ & n.s. \\
\hline HDL cholesterol $(\mathrm{mg} / \mathrm{dl})^{*}$ & $1.67(6.15)$ & $2.30(4.81)$ & n.s. & $2.67(7.64)$ & $3.72(5.71)$ & n.s. \\
\hline Systolic blood pressure $(\mathrm{mmHg})^{*}$ & $-2.07(10.67)$ & $-1.89(8.92)$ & n.s. & $-3.25(13.32)$ & $-3.21(11.26)$ & n.s. \\
\hline Diastolic blood pressure $(\mathrm{mmHg})^{*}$ & $-2.68(9.18)$ & $-3.13(7.58)$ & n.s. & $-4.21(11.29)$ & $-5.07(9.18)$ & n.s. \\
\hline
\end{tabular}

"Variation between baseline and final evaluation: presented as mean (standard deviation) and corrected for medication intensification (patients extracted from analysis: one patient from the intervention group and two from the control group, for antidiabetics; one from each group, for antihypertensives; one from intervention group, for antidyslipidemics); ${ }^{\dagger} \mathrm{p}$-values for between-groups comparisons 
Table 3.

Logistic regression examining the differences between having or not controlled glycaemia, by group.

\begin{tabular}{|c|c|c|c|c|c|c|c|c|}
\hline \multirow[t]{2}{*}{ Predictors } & \multirow{2}{*}{ B } & \multirow[t]{2}{*}{ SD } & \multirow{2}{*}{$\begin{array}{c}\chi^{2} \\
\text { Wald }\end{array}$} & \multirow[t]{2}{*}{ Df } & \multirow[t]{2}{*}{$\boldsymbol{P}$} & \multirow{2}{*}{$\begin{array}{l}\text { Odds } \\
\text { Ratio }\end{array}$} & \multicolumn{2}{|c|}{$\begin{array}{l}\mathbf{9 5 . 0 \%} \text { C.I. for Odds } \\
\text { Ratio }\end{array}$} \\
\hline & & & & & & & Lower & Upper \\
\hline Group & 1.85 & 0.82 & 5.11 & 1 & .02 & 6.35 & 1.28 & 31.56 \\
\hline Age & -0.07 & 0.03 & 4.31 & 1 & .04 & 0.93 & 0.87 & 1.00 \\
\hline Baseline glycemia & -0.03 & 0.02 & 2.81 & 1 & .09 & 0.97 & 0.93 & 1.01 \\
\hline$\% \mathbf{E W L} \mathbf{L}^{\dagger}$ & 0.02 & 0.04 & 0.35 & 1 & .55 & 1.02 & 0.95 & 1.10 \\
\hline Constant & 6.56 & 2.07 & 10.01 & 1 & .00 & 701.83 & & \\
\hline
\end{tabular}

